\title{
Correction: The outcome of fluocinolone acetonide intravitreal implant is predicted by the response to dexamethasone implant in diabetic macular oedema
}

\author{
Maria Vittoria Cicinelli $($ - Amir Rosenblatt - Domenico Grosso - Piero Zollet - Luigi Capone - Alessandro Rabiolo • \\ Rosangela Lattanzio - Anat Loewenstein - Francesco Bandello - On behalf of the International Retina Collaborative
}

Published online: 1 March 2021

(c) The Author(s), under exclusive licence to The Royal College of Ophthalmologists 2021

Correction to: Eye

$$
\text { https://doi.org/10.1038/s41433-020-01373-1 }
$$

The original version of this article unfortunately contained a mistake. The following correction has therefore been made in the original: In some instances, "edema" was changed to "oedema" and "DME" to "DMO" to be consistent throughout the article. Please find the exact instances below. The original article has been corrected.

Title: The outcome of fluocinolone acetonide intravitreal implant is predicted by the response to dexamethasone implant in diabetic macular oedema

Table 1: Duration of DMO (months)

Table 1 caption: $D M O$ diabetic macular oedema

Section "Discussion":

Systemic hypertension has been recognised as a risk factor for macular oedema in patients with diabetes [31,32]; our data suggest a worse anatomic response to intravitreal therapies in patients with high blood pressure.

On the other hand, in the setting of PDR, higher intravitreal and intraretinal levels of VEGF may maintain macular oedema, reducing the efficacy of any given antiDMO treatment.

Finally, $10 \%$ of DEX responders showed indirect signs of macular ischaemia (DRIL) [34] and nearly $80 \%$ had outer retinal disruption on SD-OCT, leading to a thinner retina when macular oedema resolved.

Section "What was known before":

- FAc implant has been approved in Europe for DMO unresponsive to previous agents.

- FAc implant yields good visual and anatomical outcomes, regardless of the nature and the number of previous DMO treatments. 